\title{
QUEDAS ENTRE CRIANÇAS E ADOLESCENTES INTERNADOS EM HOSPITAIS: REVISÃO INTEGRATIVA DE LITERATURA
}

\section{FALLS AMONG CHILDREN AND TEENAGERS IN HOSPITALS: AN INTEGRATIVE LITERATURE REVIEW}

\section{CAÍDAS ENTRE NIÑOS Y ADOLESCENTES INTERNOS EN HOSPITALES: REVISIÓN INTEGRADORA DE LITERATURA}

Gisele Lacerda Chaves Vieira ${ }^{1}$, Isa Maria Lima Campos ${ }^{2}$, Bárbara Sgarbi Morgan Fernandes ${ }^{3}$, Anne Gadelha Ladeira ${ }^{4}$, Emmanuelle Ferreira Pimenta ${ }^{5}$.

\begin{abstract}
RESUMO
Objetivo: investigar as taxas, as características, os fatores associados e as medidas preventivas relacionadas às quedas entre crianças e adolescentes em hospitais. Método: revisão integrativa de literatura realizada nas bases Scientific Electronic Library Online, Biblioteca Virtual em Saúde, National Library of Medicine e Google Scholar. Resultados: dez estudos atenderam aos critérios estabelecidos. As taxas variaram de 0.6 a 1.7 quedas por 1000 pacientes/dia. Grande parte das quedas ocorrem entre crianças do sexo masculino; com idade inferior a 9 anos. A maioria desses incidentes ocorrem na presença de um adulto. São fatores de risco para a ocorrência de quedas: idade da criança $(p<0.01)$; tempo de internação $(p<0.05)$; hiperatividade $(p<0.01)$; distúrbios hematológicos $(p<0.05)$; quedas anteriores $(p<0.01)$; dimensionamento da enfermagem $(p<0.00)$; escolaridade do cuidador $(p<0.01)$ e o fato de o mesmo ser tabagista $(p<0.01)$. As medidas preventivas adotadas foram: uso de berço e camas adequados para a idade, avaliação do risco de quedas, intervenções educacionais e uso de identificadores de alerta. Conclusão: A grande variação nas taxas de quedas entre os estudos sugere diferentes modos de organização do cuidado. A ocorrência de quedas no ambiente hospitalar entre crianças pode estar relacionada a fatores intrínsecos do paciente, do cuidador, ambientais e de organização do cuidado.
\end{abstract}

Descritores: Acidentes por quedas; Pediatria; Segurança do paciente; Criança hospitalizada; Gestão de riscos.

\section{ABSTRACT}

Objective: to investigate rates, characteristics, associated factors and preventive measures related to falls among children and adolescents in hospitals. Method: integrative literature review on the Scientific Electronic Library Online, Virtual Health Library, National Library of Medicine and Google Scholar. Results: ten studies met the established criteria. Rates ranged from 0.6 to 1.7 falls per 1000 patients / day. Most falls occur among male children; under the age of nine years old. Most of these incidents occur in the presence of an adult. Risk factors for falls are: age of the child $(p<0.01)$; length of hospital stay $(p<0.05)$; hyperactivity $(p<0.01)$; hematological disorders $(p<0.05)$; previous falls $(p<0.01)$; nursing design $(p<0.00)$; $(p<0.01)$ and the fact that it was smoker $(p$ $<0.01$ ). The preventive measures adopted were: use of cots and adequate beds for the age, evaluation of the risk of falls, educational interventions and use of alert identifiers. Conclusion: The large variation in falls rates between studies suggests different modes of care organization. The occurrence of falls in the hospital environment among children may be related to intrinsic patient, caregiver, environmental and care organization factors.

Keywords: Accidental falls; Pediatrics; Patient safety; Hospitalized children; Risk management.

\section{RESUMEN}

Objetivo: investigar las tasas, las características, los factores asociados y las medidas preventivas relacionadas con las caídas entre niños y adolescentes en hospitales. Método: revisión integrativa de literatura realizada en las bases Scientific Electronic Library Online, Biblioteca Virtual en Salud, National Library of Medicine y Google Scholar. Resultados: diez estudios atendieron a los criterios establecidos. Las tasas variaron de 0.6 a 1.7 caídas por 1000 pacientes / día. Gran parte de las caídas ocurren entre los varones; con una edad inferior a 9 años. La mayoría de estos incidentes ocurren en presencia de un adulto. Son factores de riesgo para la ocurrencia de caídas: edad del niño $(p<0.01)$; tiempo de internación ( $p<0.05)$; hiperactividad $(p<0.01)$; trastornos hematológicos ( $p<0.05)$; caídas anteriores ( $p<0.01)$; el tamaño de la enfermería $(p<0.00)$; escolaridad del cuidador $(p<0.01)$ y el hecho de que el mismo sea tabacal $(p<0.01)$. Las medidas preventivas adoptadas fueron: uso de cuna y camas adecuadas para la edad, evaluación del riesgo de caídas, intervenciones educativas y uso de identificadores de alerta. Conclusión: La gran variación en las tasas de caídas entre los estudios sugiere diferentes modos de organización del cuidado. La ocurrencia de caídas en el ambiente hospitalario entre niños puede estar relacionada a factores intrínsecos del paciente, del cuidador, ambientales y de organización del cuidado.

Descriptores: Accidentes por caídas; Pediatría; Seguridad del paciente; Niño hospitalizado; Gestión de riesgos.

${ }^{1}$ Especialista em Terapia Intensiva, Gestão da Qualidade e Segurança do Paciente. Doutora e Mestre em Enfermagem pela UFMG. ${ }^{2}$ Fisioterapeuta, Pós-Graduanda em Fisioterapia Respiratória em Terapia Intensiva. ${ }^{3}$ Doutora em Enfermagem. Coordenadora do Curso de Enfermagem do Centro Universitário Newton Paiva. ${ }^{4}$ Acadêmica do Curso de Enfermagem do Centro Universitário Newton Paiva. ${ }^{5}$ Enfermeira da Fundação Hospitalar do Estado de Minas Gerais.

Como citar este artigo:

Vieira GLC, Campos IML, Fernandes BSM, et al. Quedas entre crianças e adolescentes internados em hospitais: Revisão Integrativa de Literatura. Revista de Enfermagem do Centro Oeste Mineiro. 2019;9: e2709. [Access 


\section{INTRODUÇÃO}

As quedas entre pacientes atendidos ou internados em instituições de saúde têm sido alvo de atenção devido aos programas de segurança ao paciente instituídos em diversos países, inclusive no Brasil ${ }^{(1-3)}$. Apesar disso, grande parte das publicações e estudos sobre o tema está centrada apenas na ocorrência de quedas entre pacientes adultos ou idosos. Consequentemente, as publicações que enfocam a ocorrência de quedas entre crianças e adolescentes ainda são escassas, o que resulta em poucas informações sobre a prevalência e os fatores de risco relacionados a esse tipo de incidente ${ }^{(4)}$.

As quedas entre crianças hospitalizadas são consideradas incidentes que, por um lado, podem ser resultantes de fatores ambientais ou de riscos não identificados e/ou monitorados e também pouco abordados pela equipe assistencial, mas que, por outro lado, seriam evitáveis na maioria das situações em que ocorrem ${ }^{(5-6)}$.

A importância de se prevenir a ocorrência de quedas entre crianças, no ambiente hospitalar, deve-se ao fato de esses eventos estarem associados ao desenvolvimento de lesões e ao aumento não só do tempo de internação hospitalar como também dos custos associados à internação ${ }^{(4)}$.

Em grande parte das instituições de saúde, a redução da ocorrência de quedas constitui um dos objetivos dos cuidados de enfermagem e é monitorada como indicador associado à qualidade da assistência ${ }^{(3)}$. Para alcançar esse objetivo, é necessário conhecer os fatores de risco associados à ocorrência de quedas, o que permitirá estabelecer programas de prevenção mais efetivos dentro das instituições de saúde ${ }^{(5)}$.

O conhecimento dos fatores de risco e das estratégias preventivas no gerenciamento do risco de quedas entre crianças internadas em hospitais é o primeiro passo para se instituir medidas preventivas ${ }^{(3,5)}$. Considerando a relevância dessa temática, em especial para os profissionais de enfermagem, o objetivo deste estudo foi investigar, na literatura, as taxas, as características, os fatores associados $\mathrm{e}$ as medidas preventivas relacionadas à ocorrência de quedas entre crianças e adolescentes internados em hospitais.

\section{MÉTODOS}

Trata-se de uma pesquisa de revisão integrativa, que trata da análise e síntese de pesquisas relevantes e possibilita conclusões gerais a respeito de uma particular área de estudo ${ }^{(7)}$.

A pesquisa ocorreu durante os meses de abril a agosto de 2017. A questão norteadora desta pesquisa foi: quais são as taxas, as características, os fatores de risco e as medidas preventivas para os incidentes do tipo "quedas" entre crianças internadas em hospitais?

A pesquisa bibliográfica foi realizada nas seguintes bases de dados: BDTD (Biblioteca Digital de Teses de Dissertações), National Library of Medicine (Medline/via PubMed), BVS (Biblioteca Virtual em Saúde), Scielo (Scientific Eletronic Library Online) e Google Scholar. Foram selecionados os seguintes descritores de assunto: acidental falls; hospitalized child; hospitalized teenager; hospitals, pediatric; hospitals; child, preschool. Os termos adotados foram combinados por meio dos operadores booleanos AND e OR. Além disso, a busca dos artigos foi limitada pelo seguinte filtro: língua (inglês, português e espanhol). Optou-se por não estabelecer limites quanto ao período da publicação. Outra estratégia utilizada foi a busca manual nas listas de referências dos estudos selecionados. Para além disso, entrou-se em contato com os autores para solicitação dos estudos não disponibilizados no Portal Capes.

Além dos filtros mencionados, foram utilizados os seguintes critérios de inclusão: artigos científicos nacionais e internacionais, estudos primários, de natureza quantitativa, referentes à ocorrência de quedas entre crianças internadas em hospitais. Foram excluídos, os estudos relacionados a quedas no ambiente extra-hospitalar, entre pacientes adultos, teses e dissertações, pesquisas de abordagem qualitativa, estudos de revisão e editoriais.

As etapas de pesquisa em base de dados, seleção, análise dos estudos e coleta de dados foram realizadas de maneira independente por duas pesquisadoras. Em caso de dúvida ou discordância, solicitou-se o parecer de um terceiro revisor sobre a inclusão ou não do estudo. A coleta de dados dos artigos selecionados, por sua vez, foi realizada a partir de formulários desenvolvidos pelas autoras, com base em formulários padronizados, levando em consideração o delineamento do estudo e as recomendações estabelecidas na literatura.

Para as pesquisas de natureza quantitativa e abordagem observacional, foi utilizado o Prevalence or Incidence Critical Appraisal Instrument (Escala de Loney) ${ }^{(8)}$. Os estudos que 
obtiveram no mínimo quatro respostas afirmativas, nesse instrumento, permaneceram na amostra final da pesquisa.

Foram identificados, inicialmente, 1.482 estudos, dos quais 13 foram selecionados para leitura do texto integral. A partir da análise da qualidade desses estudos, três foram excluídos, por apresentarem limitações metodológicas importantes e pontuação na escala de Loney abaixo de quatro pontos. O fluxograma do processo de inclusão dos estudos encontra-se ilustrado na Figura 1.

Figura 1 - Descrição do processo de seleção dos artigos para resposta à questão norteadora. Belo Horizonte, MG, 2019.

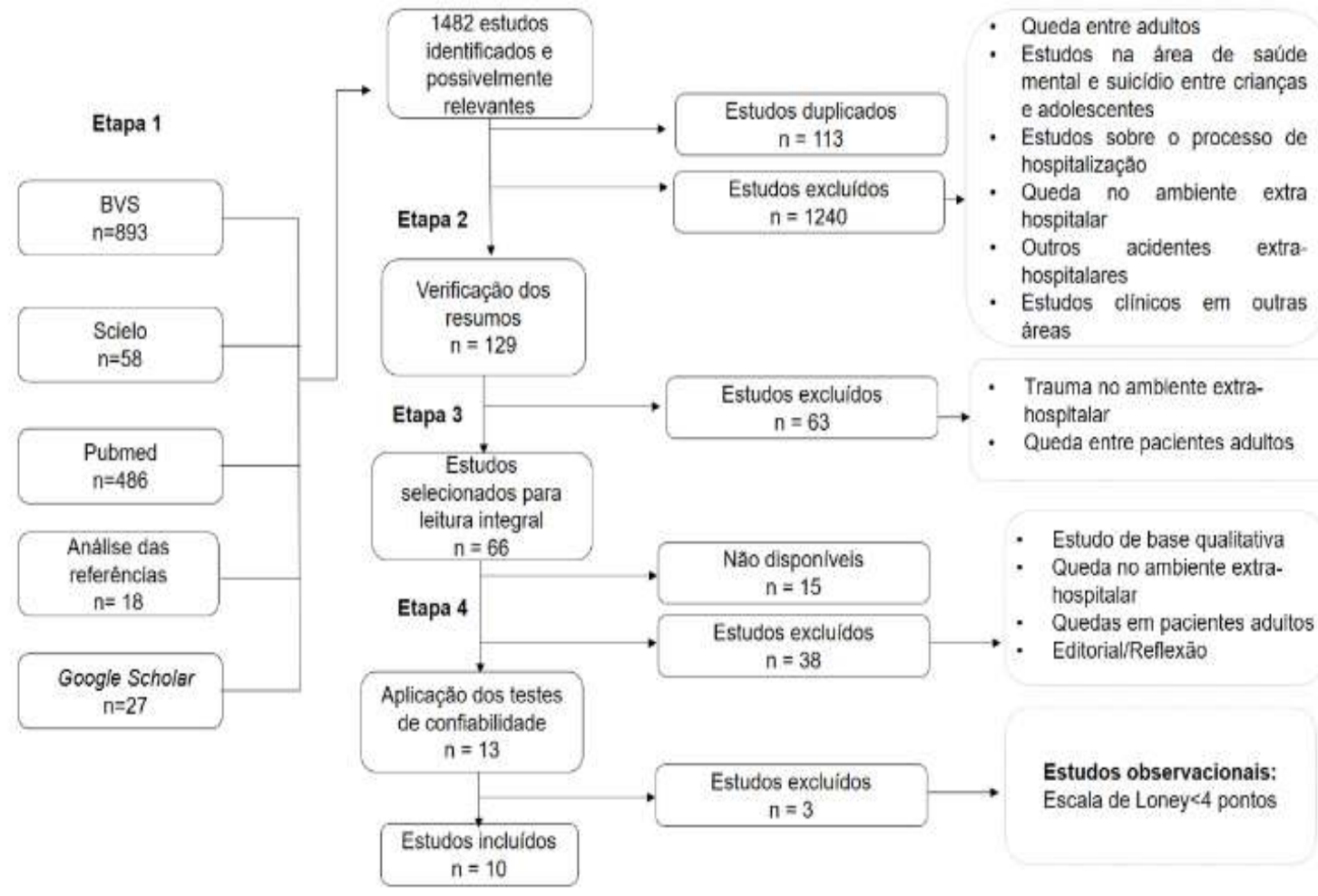

Fonte: Elaborado pelas pesquisadoras para fins deste estudo.

Com intuito de garantir a transparência no processo de revisão, contornar o viés de publicação e proteger contra os vícios de reporte, foi criado 0 protocolo de revisão noCRD42017071749, disponível no PROSPERO. Cumpre ressaltar, ainda, que os princípios éticos foram mantidos, respeitando-se os direitos autorais, mediante a citação de cada um dos autores.

\section{RESULTADOS E DISCUSSÃO}

Do total de dez artigos incluídos na amostra final, cinco estudos eram de casocontrole $^{(5,9-12)}$, quatro eram descritivos ${ }^{(2,4,13-14)}$ e um estudo de metodologia survey ${ }^{(15)}$. No que se refere à qualidade dos estudos, observa-se que nenhum obteve pontuação máxima na escala de Loney e que apenas $20.0 \% \quad(n=2)$ dos estudos obtiveram pontuação igual a $6^{(13-14)}$, o que demonstra a necessidade de se investir em pesquisas observacionais de qualidade sobre essa temática.

Todos os estudos incluídos foram publicados em língua inglesa, sendo $70.0 \%(n=7)$ realizados nos Estados Unidos ${ }^{(2,9-14)}$. Em relação ao ano de publicação, a pesquisa mais antiga foi publicada no ano de $2006^{(9)}$ e a mais atual em $2017^{(5)}$. No que se refere à prevalência de quedas entre crianças internadas no ambiente hospitalar, houve variação de 0.6 a 1.7 quedas por 1000 pacientes/dia (Figura 2$)^{(11-12)}$. 
Figura 2 - Descrição das características dos artigos que compuseram a revisão integrativa. Belo Horizonte, MG, 2019.

\begin{tabular}{|c|c|c|c|}
\hline $\begin{array}{l}\text { Autor/Ano/ } \\
\text { País/Método }\end{array}$ & Prevalência & Fatores de Risco & $\begin{array}{l}\text { Escala de } \\
\text { Loney } \\
\text { (pontos) }\end{array}$ \\
\hline $\begin{array}{l}\text { Razmus et al., } 2006 . \\
\text { Estados Unidos. Caso- } \\
\text { controle }\end{array}$ & Não explicitado & $\begin{array}{l}\text { Histórico prévio de quedas }(p<.001) ; \\
\text { presença de episódios de desorientação } \\
(p<.001)\end{array}$ & 4 \\
\hline $\begin{array}{l}\text { Hill-Rodriguez et al., } \\
\text { 2009. Estados Unidos. } \\
\text { Caso-controle. }\end{array}$ & $\begin{array}{l}0.98-1.0 \text { quedas por } 1000 \\
\text { pacientes/dia }\end{array}$ & Não avaliado & 4 \\
\hline $\begin{array}{l}\text { Kingston, Bryant, } \\
\text { Speer, 2010. Estados } \\
\text { Unidos. Descritivo. }\end{array}$ & $\begin{array}{l}\text { Hospital A: } 1.02 \text { a } 1.37 \\
\text { Hospital B: } \\
0.93 \text { a } 1.20 \\
\text { Hospital C: } \\
0.77 \text { a } 0.94 \\
\text { (quedas por } 1000 \text { pacientes/dia) }\end{array}$ & $\begin{array}{l}23 \text { a } 40 \% \text { das quedas foram relacionadas à } \\
\text { falta de atenção do familiar; } 18 \text { a } 28 \% \text { foram } \\
\text { relacionadas aos equipamentos; } 10 \text { a } 23 \% \\
\text { relacionadas às condições físicas do paciente. }\end{array}$ & 6 \\
\hline $\begin{array}{l}\text { Harvey et al., } 2010 . \\
\text { Estados Unidos. Caso- } \\
\text { controle. }\end{array}$ & $\begin{array}{l}0.6 \text { quedas/1000 pacientes/ } \\
\text { dia }\end{array}$ & $\begin{array}{l}\text { Tempo de internação }(r=0.370, p<.05) \text {; } \\
\text { hiperatividade }(r=0.458, p<.01) \text { e distúrbios } \\
\text { hematológicos }(r=0.222, p<.05) \text {. }\end{array}$ & 5 \\
\hline $\begin{array}{l}\text { Schaffer et al., } 2012 . \\
\text { Estados Unidos. } \\
\text { Descritivo. }\end{array}$ & 0.84 por 1000 pacientes/dia & Não avaliado & 4 \\
\hline $\begin{array}{l}\text { Mora et al., } 2012 . \\
\text { Itália. Descritivo. }\end{array}$ & 1.04 quedas 1000 pacientes/ dia & Não avaliado & 4 \\
\hline $\begin{array}{l}\text { Jamerson et al., } 2014 . \\
\text { Estados Unidos. } \\
\text { Descritivo. }\end{array}$ & $\begin{array}{l}0.84 \text { quedas por } 1000 \text { pacientes/ } \\
\text { dia }\end{array}$ & Não avaliado & 6 \\
\hline $\begin{array}{l}\text { Hagan J, Jones A, } \\
\text { 2015. Estados Unidos. } \\
\text { Caso-controle. }\end{array}$ & 1.7 quedas por 1000 pacientes/dia & $\begin{array}{l}\text { Menor número de profissionais de } \\
\text { enfermagem do que o recomendado para o } \\
\text { plantão }(p<.007)\end{array}$ & 5 \\
\hline $\begin{array}{l}\text { Fujita Y, Fujita M, } \\
\text { Fujiwara C, } 2013 . \\
\text { Japão. Survey. }\end{array}$ & 1,36 / 1000 pacientes-dias. & $\begin{array}{l}\text { Sala de jogos }(p<0,001) ; \text { maior relação } \\
\text { enfermeiro/enfermeiro recém-formada ( } p \\
<0,001) \text { e presença de acompanhante }(p= \\
0,04)\end{array}$ & 4 \\
\hline $\begin{array}{l}\text { Almis et al., } 2017 . \\
\text { Turquia. Caso- } \\
\text { controle. }\end{array}$ & 1.23 quedas por 1000 pacientes/dia & $\begin{array}{l}\text { Tempo de internação (OR: } 1.9 ; p<0.01 \text { ); } \\
\text { escolaridade do cuidador (OR: } 0.36 ; p<0.01 \text { ); } \\
\text { cuidador tabagista (OR: } 4.8 ; p<0.01 \text { ). }\end{array}$ & 5 \\
\hline
\end{tabular}

Fonte: Elaborado pelas pesquisadoras para fins deste estudo.

A partir do que foi apresentado na Figura 2, argumenta-se que a concentração de estudos realizados nos Estados Unidos evidencia a necessidade do desenvolvimento de pesquisas em outros países ${ }^{(2,8-11,13-14)}$. Cumpre destacar, ainda, a necessidade de se desenvolver estudos com maior qualidade metodológica a fim de produzir evidências científicas que fundamentem as práticas de prevenção de quedas em hospitais pediátricos.

No que diz respeito à existência de poucos estudos em pediatria, é possível que essa carência esteja relacionada ao fato de que as quedas que ocorrem, no caso dessa população específica, sejam consideradas pelos profissionais da área da Saúde como um evento natural, isto é, parte do desenvolvimento da criança. Tal fato, pode afetar diretamente o comportamento adotado por esses profissionais, tanto no que se refere à notificação, quanto à adoção de estratégias preventivas para a sua ocorrência.

Outro fator que pode influenciar os comportamentos dos profissionais que prestam cuidados às crianças é a não qualificação e preparo para atuar na área de segurança do paciente. Em um estudo realizado com 40 enfermeiras, $60 \%$ delas relataram não ter formação complementar na área de segurança do paciente, sendo evidenciadas fragilidades com relação às ações de registro no prontuário do resultado de avaliação e notificação de incidentes relacionados à queda entre crianças ${ }^{(16)}$. 
Ademais, o número de quedas não notificadas pelos profissionais da saúde dificulta a realização de estudos retrospectivos ${ }^{(15)}$. Grande parte das pesquisas já realizadas são de abordagem retrospectiva, o que faz com que seja necessário apontar que as taxas apresentadas nas pesquisas podem ser ainda maiores do que as evidências demonstram ${ }^{(2,4,9-11,13-15)}$.

Quando comparados a adultos, não só as taxas de quedas em pediatria são ligeiramente menores ${ }^{(11-12,16)}$, como constata-se uma variação nas taxas encontradas, que pode estar relacionada à subnotificação já mencionada e também aos diferentes modos como as estratégias preventivas são estruturadas entre as instituições. Esses fatores são corroborados por um estudo que visou comparar as taxas de quedas entre crianças internadas em três hospitais. Nesse estudo, foi verificada uma diferença estatística entre essas taxas, que, por sua vez, foi explicada pelos modos de organização do cuidado ${ }^{(13)}$.

A Figura 3 apresenta as principais características e resultados encontrados nos estudos que compuseram a amostra da presente revisão.

Figura 3 - Descrição dos objetivos e características das quedas dos artigos que compuseram a revisão integrativa. Belo Horizonte, MG, 2019.

\begin{tabular}{|c|c|c|}
\hline $\begin{array}{l}\text { Autor/Ano/ } \\
\text { País/Método }\end{array}$ & Objetivo & Características das quedas entre crianças e adolescentes \\
\hline $\begin{array}{l}\text { Razmus et al., } \\
\text { 2006. Estados } \\
\text { Unidos. Caso- } \\
\text { controle }\end{array}$ & $\begin{array}{l}\text { Identificar os fatores de risco para } \\
\text { quedas em crianças hospitalizadas, } \\
\text { utilizando duas escalas de avaliação } \\
\text { de risco de quedas validadas em } \\
\text { pacientes adultos. }\end{array}$ & $\begin{array}{l}100 \text { casos e } 100 \text { controles; } 81 \% \text { ocorreram na unidade de } \\
\text { internação; } 87 \% \text { ocorreram no quarto; } 82.8 \% \text { na presença do } \\
\text { cuidador; } 35 \% \text { caíram da cama; } 22 \% \text { ocorreram no banheiro; } \\
20 \% \text { envolveram o ato de descer ou subir na cama. }\end{array}$ \\
\hline $\begin{array}{l}\text { Hill-Rodriguez et } \\
\text { al., } 2009 . \\
\text { Estados Unidos. } \\
\text { Caso-controle. }\end{array}$ & $\begin{array}{l}\text { Avaliar a relação entre a pontuação } \\
\text { obtida na escala Humpty Dumpty e a } \\
\text { ocorrência dos eventos de queda. }\end{array}$ & $\begin{array}{l}150 \text { casos e } 152 \text { controles. Casos: } 15.7 \% \text { das quedas ocorreram } \\
\text { entre crianças menores de } 3 \text { anos, seguido de } 14.36 \% \text { entre } \\
\text { crianças com idade entre } 3 \text { e } 6 \text { anos; } 50 \% \text { dos casos eram } \\
\text { pacientes do sexo feminino; } 15.2 \% \text { tinham diagnóstico } \\
\text { associado a doenças respiratórias. }\end{array}$ \\
\hline $\begin{array}{l}\text { Kingston, Bryant, } \\
\text { Speer, } 2010 . \\
\text { Estados Unidos. } \\
\text { Descritivo. }\end{array}$ & $\begin{array}{l}\text { Comparar as taxas de quedas entre três } \\
\text { hospitais; definir uma meta anual para } \\
\text { as três instituições; definir as melhores } \\
\text { práticas para a prevenção de quedas; } \\
\text { identificar os riscos. }\end{array}$ & $\begin{array}{l}272 \text { quedas em } 2007 ; 36 \% \text { ocasionaram algum grau de dano; } 2 \% \\
\text { com dano moderado e } 98 \% \text { com dano leve. }\end{array}$ \\
\hline $\begin{array}{l}\text { Harvey et al., } 2010 . \\
\text { Estados Unidos. } \\
\text { Caso-controle. }\end{array}$ & $\begin{array}{l}\text { Avaliar os instrumentos de queda } \\
\text { disponíveis e construir um modelo } \\
\text { preditivo para o risco de lesão e } \\
\text { ocorrência de queda. }\end{array}$ & $\begin{array}{l}99 \text { crianças, sendo } 33 \text { casos e } 66 \text { controles, } 63.4 \% \text { com idade } \\
\text { entre } 5 \text { e } 9 \text { anos. Queda mais comum entre 3-8 dias após a } \\
\text { admissão. }\end{array}$ \\
\hline $\begin{array}{l}\text { Schaffer et al., } \\
\text { 2012. Estados } \\
\text { Unidos. Descritivo. }\end{array}$ & $\begin{array}{l}\text { Identificar as características dos } \\
\text { pacientes e os fatores ambientais } \\
\text { relacionados aos danos associados às } \\
\text { quedas em hospitais pediátricos. }\end{array}$ & $\begin{array}{l}53 \text { quedas; } 58.5 \% \text { com dano; } 17 \% \text { necessitaram de tratamento } \\
\text { médico; } 83 \% \text { das crianças com desenvolvimento apropriado } \\
\text { para a idade; } 58 \% \text { no quarto; a idade variou de } 4 \text { meses a } 17 \\
\text { anos, } 58.5 \% \text { do sexo masculino; } 52.8 \% \text { sem histórico prévio de } \\
\text { queda e } 84.9 \% \text { sem convulsão; } 98 \% \text { encontravam-se alertas, } \\
24.5 \% \text { abaixo de } 3 \text { anos; } 69.8 \% \text { na presença de um adulto. }\end{array}$ \\
\hline $\begin{array}{l}\text { Mora et al., } 2012 . \\
\text { Itália. Descritivo. }\end{array}$ & $\begin{array}{l}\text { Avaliar o número de quedas entre } \\
\text { crianças admitidas em um hospital e } \\
\text { explorar as características das } \\
\text { quedas. }\end{array}$ & $\begin{array}{l}58 \text { quedas; } 55.2 \% \text { entre idade de } 1 \text { a } 3 \text { anos; } 56.9 \% \text { do sexo } \\
\text { masculino; } 55.2 \% \text { entre o final da tarde até meia-noite; } 39.6 \% \\
\text { como causa a perda de equilíbrio; } 58.6 \% \text { no quarto do paciente; } \\
91.4 \% \text { na presença dos pais; } 32.8 \% \text { enquanto as crianças } \\
\text { estavam andando; } 31 \% \text { envolveram queda do berço; } 51.7 \% \text { não } \\
\text { envolveram causas comportamentais ou ambientais; } 98,2 \% \\
\text { apresentaram lesão decorrente. }\end{array}$ \\
\hline $\begin{array}{l}\text { Jamerson et al., } \\
\text { 2014. Estados } \\
\text { Unidos. Descritivo. }\end{array}$ & $\begin{array}{l}\text { Compreender a natureza das quedas } \\
\text { na pediatria, a sua prevalência, } \\
\text { identificar a ocorrência de danos, } \\
\text { característica das quedas associadas } \\
\text { com lesão. }\end{array}$ & $\begin{array}{l}782 \text { quedas, } 32 \% \text { apresentaram lesão; } 48 \% \text { classificadas como } \\
\text { preveníveis; } 64 \% \text { no quarto e } 13 \% \text { no banheiro; } 77 \% \text { na } \\
\text { presença de um adulto; } 52 \% \text { da cama/berço/maca; } 54 \% \text { são } \\
\text { pacientes do sexo masculino; em } 86 \% \text {, o desenvolvimento era } \\
\text { apropriado para a idade; } 66 \% \text { deambulavam de forma } \\
\text { independente; } 70 \% \text { apresentavam marcha estável; } 32 \% \text { com } \\
\text { média de idade de } 6 \text { anos }\end{array}$ \\
\hline $\begin{array}{l}\text { Hagan J, Jones A, } \\
\text { 2015. Estados } \\
\text { Unidos. Caso- } \\
\text { controle. }\end{array}$ & $\begin{array}{l}\text { Examinar a relação entre o } \\
\text { dimensionamento de enfermagem e } \\
\text { a ocorrência de quedas. }\end{array}$ & $\begin{array}{l}111 \text { quedas; } 36 \% \text { com dano; } 19 \% \text { na unidade de internação; } 26 \% \\
\text { com crianças que possuíam um dos seguintes diagnósticos: } \\
\text { convulsão; atraso no desenvolvimento motor; confusão mental; } \\
\text { marcha instável. }\end{array}$ \\
\hline
\end{tabular}




\begin{tabular}{|l|l|l|}
\hline $\begin{array}{l}\text { Fujita Y, Fujita M, } \\
\text { Fujiwara C, 2013. } \\
\text { Japão. Caso- } \\
\text { controle. }\end{array}$ & $\begin{array}{l}\text { Identificar o efeito de medidas de } \\
\text { prevenção de quedas e características } \\
\text { das enfermarias sobre a taxa de } \\
\text { quedas pediátricas. }\end{array}$ & $\begin{array}{l}162 \text { quedas; os autores não apresentaram as características das } \\
\text { quedas devido à indisponibilidade destas informações. }\end{array}$ \\
\hline $\begin{array}{l}\text { Almis et al., 2017. } \\
\text { Turquia. Caso- } \\
\text { controle. }\end{array}$ & $\begin{array}{l}\text { Avaliar os fatores de risco para } \\
\text { quedas, relacionados aos cuidadores, } \\
\text { em crianças hospitalizadas. }\end{array}$ & $\begin{array}{l}39 \text { casos e } 78 \text { controles. Casos: } 59 \% \text { eram do sexo masculino, } \\
\text { com média de idade de } 14.7 \text { meses; tempo médio de internação } \\
\text { de } 5.8 \text { dias; } 71.8 \% \text { dos cuidadores possuíam nível de } \\
\text { escolaridade de até } 5 \text { anos; média de idade de } 29.3 \text { anos; sendo } \\
94.9 \% \text { donas de casa; } 23.1 \% \text { eram tabagistas; } 2 \text { pacientes } \\
\text { sofreram danos graves. O maior número de quedas ocorreu no } \\
\text { dia da admissão. }\end{array}$ \\
\hline
\end{tabular}

Fonte: Elaborado pelas pesquisadoras para fins deste estudo.

Quanto às características das crianças, em grande parte das pesquisas, houve maior porcentagem de ocorrência desse tipo de incidente entre o sexo masculino ${ }^{(2,4-5,14)}$ e com idade inferior a 9 anos ${ }^{(4-5,10-11)}$. Em alguns estudos, as quedas foram mais preponderantes entre crianças abaixo de 3 anos de idade ${ }^{(4,5)}$. Em relação ao local, grande parte das quedas ocorreu no quarto do paciente, podendo ser uma queda da cama ou berço $o^{(2,4,9,14)}$. Já para as quedas entre adolescentes, grande parte ocorreu no banheiro ${ }^{(9,14)}$. Por fim, no que tange aos estudos que relataram ocorrência de dano, observou-se variação de $17 \%$ a $98.2 \%^{(2,4,12-14)}$. Apenas um estudo analisou se as quedas eram preveníveis ou não, sendo $48 \%$ delas consideradas preveníveis (Figura 3) $^{(14)}$.

As crianças com menor idade são consideradas as mais vulneráveis, por se encontrarem em um ambiente desconhecido e não realizarem julgamento dos riscos aos quais estão expostas ${ }^{(4)}$. Da mesma maneira, as crianças do sexo masculino apresentam maior tendência a apresentar um comportamento mais agitado e hiperativo ${ }^{(13)}$. Por esses motivos, diversos autores defendem a necessidade de se considerar todas as crianças que possuem um comportamento agitado/agressivo ou que possuam déficits de atenção, como apresentando alto risco para quedas no ambiente hospitalar ${ }^{(11)}$.

No entanto, é importante ressaltar ainda, não só que é possível observar diferentes aspectos relacionados à ocorrência de quedas no ambiente hospitalar que não foram retratados nas pesquisas analisadas, como também que não foi encontrada uma pesquisa que descreva, de forma completa, as circunstâncias em que as quedas ocorreram. Sendo assim, considera-se que os resultados evidenciam a necessidade de mapeamento desses riscos, bem como de estabelecimento de estratégias multifatoriais de prevenção desses incidentes.
Com relação aos fatores de risco para a ocorrência de quedas, verificou-se o tempo de internação $(p<0.05)^{(11)}$ como um dos principais, sendo que o risco é maior no dia da admissão e tende a aumentar novamente a partir de três dias internação. Além disso, há maior risco de quedas entre crianças hiperativas $(p<0.01)$ e com distúrbios hematológicos $(p<0.05)^{(11)}$. Para esse último, ressalta-se a baixa correlação encontrada $(r=0.22)$. O histórico prévio de quedas $(p<0.01)$ e a presença de episódios de desorientação $(p<0.01)$ também foram verificados como fatores de risco para novos incidentes ${ }^{(9)}$.

Em contrapartida, o dimensionamento de recursos humanos na enfermagem $(p<0.00)$, juntamente com uma maior proporção desses profissionais possuindo pouca experiência $(p<0.00)$, apresentou associação significativa com a ocorrência de quedas na pediatria ${ }^{(15)}$. Em um dos estudos, por outro lado, foi encontrada correlação significativa entre a ocorrência de quedas e a sala de brinquedos $(p<0.001)^{(15)}$, enquanto outro estudo apresentou associação entre o risco de queda com a escolaridade do cuidador $(p<0.01)$ e o fato de esse profissional ser tabagista $(p<0.01)$ (Figura 3$)^{(5)}$.

Apenas quatro estudos abordaram as medidas preventivas em relação à ocorrência de quedas na pediatria ${ }^{(9,13-15)}$. As medidas recomendadas mais frequentemente foram: 1) uso de berço e camas adequados para a idade; 2 ) avaliação do risco de quedas sempre que houver mudanças no quadro clínico, transferência entre setores ou realização de procedimentos cirúrgicos; 3) instrução dos cuidadores a cada troca de plantão; 4) supervisão próxima de crianças com alto risco de quedas; 5) uso de identificadores que alertem quanto ao alto risco de quedas e 6) capacitação dos profissionais recém admitidos na instituição ${ }^{(9,13-15)}$.

Apesar de existirem fatores claramente definidos relacionados à ocorrência de quedas no ambiente hospitalar em adultos, entre crianças 
há muito ainda a ser investigado ${ }^{(5)}$. Na pediatria, observa-se que as quedas podem estar relacionadas a fatores intrínsecos ao paciente (condições clínicas, personalidade e comportamento); a fatores ambientais (organização do espaço na enfermaria, luminosidade e sinalização) ${ }^{(13,15)}$; a fatores relacionados à organização do cuidado (dimensionamento dos profissionais de enfermagem, uso de estratégias e protocolos de prevenção e capacitação dos profissionais) $)^{(12,15)}$ e a fatores relacionados ao acompanhante (idade, escolaridade, tabagismo, cansaço) ${ }^{(5)}$.

Além disso, enquanto entre adultos, esse último fator pode não ser o de maior influência, entre crianças a presença de um adulto pode ser o fator definidor para a prevenção de quedas no ambiente hospitalar ${ }^{(5)}$.

Tal fato reforça a importância de se instituir estratégias educacionais que envolvam os profissionais da saúde e os pais e/ou acompanhantes das crianças internadas ${ }^{(15)}$, uma vez que a maioria dos incidentes encontrados, neste estudo, ocorreu na presença de um adulto $^{(2,4,9,14)}$.

Sugere-se, assim, que parâmetros adicionais sejam incluídos em escalas que avaliem o risco de quedas em crianças, com acréscimo de itens relacionados ao acompanhante e aos aspectos comportamentais da criança. Entretanto, cumpre esclarecer que nenhuma escala pode substituir o gerenciamento do risco à beira do leito, com a supervisão atenta dos profissionais de enfermagem e dos acompanhantes dessas crianças ${ }^{(11)}$.

Associado à necessidade de se investir na formação dos profissionais de saúde, o dimensionamento dos profissionais de enfermagem deve ser considerado como fator contribuinte à ocorrência de eventos adversos entre pacientes hospitalizados. Ademais, sabe-se que o quantitativo de profissionais é fator contribuinte para a qualidade da assistência, apesar de terem sido encontrados apenas dois estudos que tratassem sobre essa temática no âmbito da pediatria ${ }^{(12,15)}$. Para além disso, o subdimensionamento dos profissionais de enfermagem e as duplas jornadas de trabalho são fatores que influenciam diretamente na qualidade da assistência ${ }^{(18)}$ e que se encontram relacionadas à ocorrência de quedas entre crianças internadas ${ }^{(12,15)}$.

Embora existam diferenças metodológicas entre as pesquisas, estratégias preventivas para a ocorrência de quedas puderam ser encontradas conforme os fatores de risco identificados entre os pacientes. Abordagens para a mudança da cultura de segurança relacionadas a programas de prevenção de quedas ainda são recentes na literatura. A avaliação do risco de queda para cada paciente internado é o passo inicial para o gerenciamento desse risco. No entanto, as escalas disponibilizadas para a avaliação do risco de quedas em pediatria não apresentam confiabilidade adequadas para serem utilizadas $^{(11)}$. Dessa forma, argumenta-se que uma estratégia interessante seria estabelecer intervenções para todos os pacientes hospitalizados.

De forma complementar, se os profissionais de saúde se conscientizarem das possíveis razões para as quedas que ocorrem no ambiente hospitalar, será possível, então, mapear os riscos aos quais as crianças estão expostas e implementar estratégias preventivas. Essas ações devem ser direcionadas para a melhoria de todos os processos relacionados ao cuidado do paciente, uma vez que são diversos os fatores de risco para a ocorrência das quedas e que é necessário o uso de medidas e protocolos institucionais a fim de mitigar esses riscos e reduzir a ocorrência desse tipo de incidente. Em um dos estudos escolhidos para integrar esta revisão, verificou-se que metade da variância das quedas, à análise dos dados por meio de regressão logística, puderam ser explicadas pela organização do cuidado e fatores ambientais da instituição ${ }^{(15,19)}$.

Ao mesmo tempo, como parte representativa das quedas envolve o berço ou a cama em que a criança se encontra, adverte-se para a importância de se instituir medidas preventivas relacionadas à adequação do berço em que as crianças são colocadas durante a internação. O uso de critérios para a seleção de berços adequados para as crianças pode ser associado à menor ocorrência de quedas entre essa população. Ressalta-se ainda que, se as crianças são capazes de subir nas grades dos berços, quando possuírem altura superior a 90 $\mathrm{cm}$, devem ser colocadas em camas e não mais em berços. No entanto, apesar de haver algumas recomendações no que se refere ao uso de berços adequados para o tamanho da criança, no Brasil, ainda não existem legislações claras que tratem sobre o assunto, o que evidencia a necessidade de se realizar pesquisas sobre essa questão ${ }^{(15)}$. 
Quanto ao envolvimento dos pais e/ou acompanhantes no gerenciamento do risco de quedas, deve-se dar atenção especial ao momento em que eles recebem orientações dos profissionais da saúde e também à regularidade em que essas informações são repassadas a eles. No momento da admissão é possível que, ao receber as informações, os pais e/ou acompanhantes das crianças estejam demasiadamente estressados para compreender tudo o que lhes é repassado. Além disso, o número de quedas tende a aumentar com o tempo de internação, o que sugere a necessidade de reforço durante o processo educacional ${ }^{(15)}$.

Sabe-se ainda que, na maioria das vezes, há um rodízio entre os acompanhantes, o que evidencia a necessidade de que os processos educacionais para o gerenciamento do risco de queda sejam repassados continuamente aos acompanhantes, enquanto as crianças estiverem internadas. À medida que o tempo de internação aumenta, os pais e/ou acompanhantes também apresentam maior nível de estresse e cansaço, o que pode influenciar a ocorrência das quedas na pediatria $^{(5,15)}$.

Outro fator que pode influenciar a ocorrência dessas quedas é a baixa escolaridade dos pais e/ou acompanhantes. É possível que pais com baixa escolaridade tenham maior dificuldade de compreender as informações que lhe são repassadas ou a sua importância. Esses fatores também evidenciam a importância de se atentar para a forma como essas informações são repassadas pelos profissionais da saúde aos acompanhantes. Nesse sentido, diversos autores argumentam sobre a importância da utilização de multiestratégias no processo educacional dos cuidadores, como o uso de folhetos associados às orientações verbais ${ }^{(15,17)}$.

Como limitação para este estudo, ressaltase a escassez de publicações relacionadas à temática e a inexistência de estudos com alta qualidade metodológica. A fim de minimizar essas limitações, instituiu-se a análise de qualidade das pesquisas encontradas, sendo excluídos os estudos que apresentaram pontuação inferior à previamente estabelecida. Cumpre enfatizar, ainda, a necessidade de se realizar estudos observacionais que investiguem os fatores de risco associados à ocorrência de quedas entre crianças internadas em hospitais pediátricos. A realização desses estudos poderá auxiliar na constituição de medidas preventivas adequadas à essa população.

\section{CONCLUSÃO}

Apesar de haver uma escassez de estudos sobre quedas entre crianças internadas em unidades de pediatria, foi possível identificar que as taxas de quedas, nessa população, são inferiores quando comparadas às taxas entre pacientes adultos, variando entre 0.6 a 1.7 quedas por 1000 pacientes/dia.

No que diz respeito às características e aos fatores associados às quedas, essas podem ser caracterizadas e/ou associadas com o local e a circunstância de ocorrência, as características do ambiente e as características do acompanhante. As quedas estão relacionadas a múltiplos fatores como idade, sexo, características clínicas e de personalidade da criança, bem como à organização do cuidado e ao dimensionamento do pessoal de enfermagem

Como proposta subsequente a este estudo, pretende-se desenvolver uma pesquisa observacional do tipo caso-controle, a fim de identificar os fatores de risco, as causas e as circunstâncias relacionadas à ocorrência de quedas entre crianças internadas em um hospital pediátrico.

\section{REFERÊNCIAS}

1 - Brasil. Ministério da Saúde. Protocolo prevenção de quedas. Brasil: Ministério da Saúde; 2013.

2 - Schaffer PL, Daraiseh NM, Daum L, Mendez E, Lin L, Huth MM. Pediatric inpatient falls and injuries: A descriptive analysis of risk factors. J Spec Pediatr Nurs. 2012;17(1):10-8. DOI: 10.1111/i.1744-6155.2011.00315.x

3 - Gu YY, Balcaen K, Ni Y, Ampe J, Goffin J. Review on prevention of falls in hospital settings. CNR 2016;3(1):7-10. DOI: 10.1016/j.cnre.2015.11.002

4 - Mora DRD, Bagnasco A, Sasso L. In-hospital paediatric acidentes: An integrative review of the literature. Int Nurs Rev. 2012;59(4):466-73. DOI: 10.1111/i.1466-7657.2012.01015.x

5 - Almis H, Bucak HH, Konca C, Turgut M. Risk factors related to caregivers in hospitalized children's falls. J Pediatr Nurs. 2017;32:3-7. DOI: 10.1016/i.pedn.2016.10.006

6 - Torino VV, Tsunechiro MA, Santos AU, Aragaki IMM, Shimoda GT. Queda de recém-nascido internado em alojamento conjunto. Cogitare Enferm. 2016;21(4):1-8. DOI: 10.5380/ce.v21i4.45852

7 - Mendes KDS, Silveira RCCP, Galvão CM. Revisão integrativa: Método de pesquisa para a 
incorporação de evidências na saúde e na enfermagem. Texto Contexto-Enferm. 2008;17(4):758-764. DOI: $10.1590 /$ S010407072008000400018

8 - Loney PL, Chambers LW, Bennett KJ, Roberts JG, Stratford PW. Critical appraisal of the health research literature: Prevalence or incidence of a health problem. Chonic Dis Can. 1998 [citado em 15 nov 2018]; 19(4):170-6. Disponível em: https://www.ncbi.nlm.nih.gov/pubmed/10029513 9 - Razmus I, Wilson D, Smith R, Newman E. Falls in hospitalized children. Pediatr Nurs. 2006 [citado em 15 nov 2018]; 32(6):568-72. Disponível em:

https://www.ncbi.nlm.nih.gov/pubmed/17256296 10 - Hill-Rodriguez D, Messner PR, Williams PD, Zeller RA, Williams AR, Wood $M$, et al. The humpty dumpty falls scale: A case-control study. J Spec Pediatr Nurs. 2009;14(1):22-32. DOI: 10.1111/j.1744-6155.2008.00166.x

11 - Harvey K, Kramlich D, Chapman J, Parker J, Blades E. Exploring and evaluating five pediatric falls assessment instruments and injury risk indicators: An ambispective study in a tertiary care setting. J Nurs Manag. 2010;18(5):531-41. DOI: 10.1111/j.1365-2834.2010.01095.x

12 - Hagan J, Jones A. Lower nurse staffing levels are associated with occurrences of inpatient falls at a large pediatric hospital. Health Care Manag. 2015;34(4):359-66.

DOI: 10.1097/HCM.0000000000000083

13 - Kingston F, Bryant T, Speer K. Pediatric falls benchmarking collaborative. J Nurs Adm. 2010;40(6):287-92.

DOI: 10.1097/NNA.0b013e3181df10d9

14 - Jamerson PA, Graf E, Messner PR, Fields HW, Barton $S$, Berger $A$, et al. Inpatient falls in freestanding children's hospitals. Pediatr Nurs. 2014 [citado em 15 nov 2018]; 40(3):127-35. Disponível em: https://www.ncbi.nlm.nih.gov/pubmed/25134226 15 - Fujita Y, Fujita M, Fujiwara C. Pediatric falls: Effect of prevention measures and characteristics of pediatric wards. Jpn J Nurs Sci. 2013;10(2):22331. DOI: $10.1111 / \mathrm{jins} .12004$

16 - Gurgel SS, Ferreira MKM, Sandoval LS, Araújo PR, Galvão MTG, Lima FEIT. Competências do enfermeiro na prevenção de quedas em crianças à luz do consenso de Galway. Texto Contexto-Enferm. 2017;26(4):1-9. DOI: 10.1590/0104-070720170003140016

17 - Luzia MF, Victor MAG, Lucena AF. Diagnóstico de enfermagem risco de quedas: prevalência e perfil clínico de pacientes hospitalizados. Rev Latino-Am Enfermagem 2014;22(2):262-8. DOI: 10.1590/0104$\underline{1169.3250 .2411}$

18 - Cho E, Chin DL, Kim S, Hong O, Faaohn F. The relationships of nurse staffing level and work environment with patient adverse events. J Nurs Scholarsh. 2015;48(1):74-82. DOI: 10.1111/inu.12183

19 - Kramlich DL, Dende D. Development of a pediatric fall risk and injury reduction program. Pediatr Nurs. 2016 [citado em 15 out 2018]; 42(2):77-82. Disponível em: https://www.ncbi.nlm.nih.gov/pubmed/27254976

Nota: Este artigo é parte de relatório de pesquisa de iniciação científica do Centro Universitário Newton Paiva e da Fundação Hospitalar do Estado de Minas Gerais. Fomento: Programa de Iniciação Científica do Centro Universitário Newton Paiva.

Recebido em: 26/12/2017

Aprovado em: 08/04/2019

Endereço de correspondência:

Gisele de Lacerda Chaves Vieira

Avenida Silva Lobo, 1730 - Grajaú

CEP: 30.431-262 - Belo Horizonte/MG - Brasil

E-mail: giselelacerdavi@gmail.com 